\title{
Nivel de cumplimiento de las fases del tratamiento periodontal en las Clínicas Odontológicas de la Universidad de Santo Tomás
}

\author{
Level of compliance of the phases of periodontal treatment in the Dental Clinics \\ of the University of Santo Tomás
}

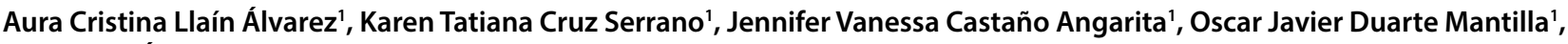
Jhon Jairo Álvarez Martínez²
\end{abstract}

Para citar este artículo: Llaín-Álvarez AC, Cruz-Serrano KT, Castaño-Angarita JV, Duarte-Mantilla OJ, Álvarez-Martínez JJ. Nivel de cumplimiento de las fases del tratamiento periodontal en las Clínicas Odontológicas de la Universidad Santo Tomás. Ustasalud 2016;15:20-26.

Licencia Creative Commons

(c) (1) (5)
lo tanto, los lectores pueden acceder libremente a los artículos en su formato .pdf, igualmente podrán descargarlos y difundirlos; sin embargo no podrán modificarlos o alterarlos, adicionalmente se debe reconocer la autoría de las personas que figuran en las publicaciones, pero estas no podrán comercializadas.

1 Estudiante de Odontología, Universidad Santo Tomás, Bucaramanga.

2 Docente Facultad de Odontología, Universidad Santo Tomás, Bucaramanga

Autor de correspondencia: Jhon Jairo Álvarez Martínez Correo electrónico: john.alvarez@ustabuca.edu.co

Recibido para publicación: 08 de marzo de 2016 Aceptado para publicación: 26 de julio de 2016.

\section{RESUMEN}

Objetivo: determinar el nivel de cumplimiento de las fases del tratamiento periodontal registrado en las historias clínicas de pacientes que atienden a las Clínicas Odontológicas de la Universidad Santo Tomás, Bucaramanga durante el 2016.

Materiales y métodos: se realizó un estudio observacional de corte transversal, se tuvieron en cuenta 171 historias clínicas que cumplían todos los criterios de selección, se evaluaron variables sociodemográficas, semestre del estudiante que atendía al paciente, compromiso sistémico, diagnóstico periodontal, y registro de las fases del tratamiento periodontal.

Resultados: la fase de mantenimiento tuvo mayor nivel de incumplimiento $(99,4 \%)$, seguida de la fase de reevaluación $(98,8 \%)$. La fase higiénica presentó un incumplimiento del $57,3 \%$. Dentro de las fases que requieren o no de su cumplimiento; la fase urgencia, evidenciada por dos personas, solo una de estas cumplió; en la fase sistémica, el $69,6 \%$ asistió a la consulta para el tratamiento periodontal y en la fase correctiva, se presentó un incumplimiento del 78,9\%.

Conclusión: el estudiante debe recalcar en cada atención al paciente la importancia de cumplir con cada una de las fases del tratamiento periodontal, especialmente, con la asistencia a las fases de reevaluación y mantenimiento, ya que se evidenció en estas un alto porcentaje de incumplimiento.

Palabras clave: índice periodontal, periodontitis crónica, enfermedad periodontal, furca.

\section{ABSTRACT}

Objective: to determine the level of compliance of the phases of periodontal treatment in patients attending the Dental Clinics of the Universidad Santo Tomás, located in Floridablanca and Bucaramanga during the academic year of 2016.

Materials and methods: a cross-sectional study, 171 clinical histories were considered that met all the selection criteria. Socio-demographic variables, semester, systemic commitment, periodontal diagnosis, periodontal treatment phases were evaluated.

Results: the maintenance phase had a higher level of noncompliance (99.4\%), followed by the reassessment phase $(98.8 \%)$, for the phases that, depending on the course of the disease, are required to be required or not; The urgency phase, demanded by two people had a non-compliance of 50\%; In the systemic phase, $69.6 \%$ attended the consultation for periodontal treatment and for the corrective phase, there was a high level of noncompliance of $78.9 \%$ for the required phases; In similarity, the hygienic phase presented a high level of non-compliance: $57.3 \%$.

Conclusion: probably, the causes of high failure rates of periodontal treatment are due to weak strategies of education and motivation for the patient. The importance of the results of this study is to allow to base the creation, redesign and strengthening of interventions in the consultations to insist to the patient on the importance of assisting in the phases of reevaluation and maintenance since it was evidenced in these two phases a high Percentage of default.

Keywords: periodontal index, chronic periodontitis, periodontal disease, furca. 


\section{INTRODUCCIÓN}

Las enfermedades periodontales son condiciones infecciosas-inflamatorias crónicas que afectan a los tejidos de soporte y protección del diente ${ }^{1}$. Las entidades más comunes encontradas son la gingivitis y la periodontitis, siendo estas causadas por bacterias que se encuentran en el biofilm² ${ }^{2}$.

La terapia de la enfermedad periodontal ha ido evolucionando a lo largo de los años. En la década de los cincuenta, la periodontitis era tratada principalmente mediante la extracción de los dientes afectados. A finales de los setenta y comienzo de los ochenta, se realizaban cirugías a campo abierto siendo la ausencia o presencia de bolsas lo que determinaba el éxito o no de la terapia ${ }^{3}$. Actualmente el objetivo de la terapia periodontal es conservar los tejidos periodontales sanos y los dientes biológicamente aceptables; para lograrlo se debe seguir una estrategia terapéutica la cual se basa en el control de los factores etiológicos locales que implican riesgo en la progresión de la lesión ${ }^{4,5}$.

El examen periodontal básico (EPB) es una pauta muy útil que ha sido recomendada para ser usada en la práctica odontológica para registrar los hallazgos periodontales y diagnosticar las periodontopatías. Es importante que todos los pacientes sean examinados y que todos los odontólogos estén atentos a cualquier signo de irritación periodontal ${ }^{6}$.

El plan de tratamiento periodontal es la pauta para el manejo de casos, aquí se incluyen todos los procedimientos necesarios para el establecimiento y mantenimiento de la salud gingival. Se realiza en una secuencia de fases que pueden variar dependiendo de las necesidades de cada caso ${ }^{7}$.

Las fases del tratamiento periodontal necesitan de diferentes parámetros para su cumplimiento; uno de ellos es la asistencia a las citas odontológicas, en las que se realizan los procedimientos de acuerdo con la periodontopatía encontrada en cada paciente.

A nivel nacional y regional no existe ningún estudio donde se verifique la eficacia en el cumplimiento de las fases del tratamiento periodontal que se debe aplicar a pacientes que presenten enfermedad, a pesar de que la enfermedad periodontal es una de las entidades más frecuentes a nivel mundial, una de las principales causas de pérdida dentaria y es considerada un problema de salud pública ${ }^{8}$.

Se hizo necesario el desarrollo de esta investigación para verificar el nivel de cumplimiento de las fases del tratamiento periodontal, basándonos en los conocimientos adquiridos y por medio de la literatura en la cual se determinan una serie de procedimientos necesarios para cada una de las fases y con base en esto evaluar las falencias académicas que hacen un indebido manejo periodontal. Este trabajo servirá como referente institucional para observar qué fases del tratamiento periodontal se ejecutan, cuál es la de mayor cumplimiento y hacer que se cumplan correctamente a fin de generar estrategias para conseguir el $100 \%$ de cumplimiento en el tratamiento periodontal.

Con la información anteriormente expuesta, el objetivo de esta investigación es determinar el nivel de cumplimiento de las fases del tratamiento periodontal en pacientes que asisten a las Clínicas Odontológicas de la Universidad Santo Tomás Campus Floridablanca y Bucaramanga durante el año académico 2016. Así mismo verificar cuál de las fases del tratamiento periodontal tiene mayor cumplimiento y evaluar la asistencia de los pacientes a las fases de reevaluación y mantenimiento.

\section{MATERIALES Y MÉTODOS}

Se realizó un estudio observacional descriptivo de corte transversal. En la presente investigación se tuvieron en cuenta la Ley Estatutaria 1581 de 2012 y la Resolución 008430 del 1993 del Ministerio de Salud de la República de Colombia, en las cuales se cataloga esta investigación como sin riesgo; debido a que no provocó ningún daño físico, ni sufrimiento psicológico, ya que se realizó a partir de los datos disponibles en las historias clínicas.

La población estuvo conformada por las historias clínicas de pacientes que asistieron a las Clínicas Odontológicas de pregrado de la Universidad Santo Tomás, durante un año lectivo. Solo se tuvieron en cuenta las historias clínicas que tenían diligenciado el consentimiento informado exclusivo de investigación. 
La población estuvo conformada por 171 historias clínicas que fueron diligenciadas por estudiantes de pregrado de sexto a décimo semestre, aprobadas y firmadas por el docente de módulo y periodontogramas que indicaron enfermedad periodontal y necesidad de tratamiento. Las historias clínicas fueron prestadas bajo acta; se tuvieron en cuenta las medidas de bioseguridad y las historias clínicas fueron manipuladas con cuidado por los investigadores, minimizando el impacto físico del documento y el riesgo del investigador.

Para la realización de la prueba piloto se utilizaron 10 historias clínicas, revisando el plan de tratamiento periodontal y su ejecución en la evolución de las actividades clínicas. Se comenzó el proceso de recolección de datos con las historias clínicas de archivo pasivo, extrayendo bloques de 80 unidades que fueron examinadas en la Oficina de Archivo, ya que es el lugar indicado y cuenta con el espacio y el mobiliario apropiado para dicha tarea.

El análisis estadístico se realizó con el software Stata Versión $14.0^{9}$. Para las variables cuantitativas se calcularon promedios y se halló la mediana en caso de no encontrarse una distribución normal; para el caso de las variables cualitativas se calcularon proporciones y frecuencias.

Si bien la clasificación de la enfermedad periodontal es extensa y se confirma en el International Work-shop for a Clasification of Periodontal Disease and Conditions, en $1999^{10}$. Este estudio solo se enfocó en el cumplimiento de las fases del tratamiento periodontal. La planificación del tratamiento periodontal se compone de una secuencia de medidas terapéuticas usadas para controlar la enfermedad periodontal, para cumplir con este fin, el plan de tratamiento periodontal debe desglosarse en las siguientes fases:

Fase de urgencia: se encarga del alivio del dolor y control de inflamación aguda. Dependiendo de la sintomatología y estado sistémico se formulan medicamentos.

Fase sistémica: revisión del estado de salud general del paciente, se analiza la situación antes de iniciar tratamiento, en caso de que exista compromiso sistémico se debe realizar interconsulta médica.
Fase higiénica: su objetivo es el control de factores locales por medio de terapia periodontal básica (no quirúrgica), se realiza: control de placa, fisioterapia oral, detartraje supragingival, profilaxis, raspado y alisado radicular a campo cerrado, terapia química, operatoria, endodoncia, exodoncia.

Fase reevaluación: se ejecuta a los 30 días de haber finalizado la fase higiénica. Su objetivo, valorar la respuesta de los tejidos periodontales, si se logró salud periodontal, se fijan intervalos de mantenimiento, si no se obtuvo, se realizan de nuevo los procedimientos; se hace control de placa y se toman decisiones.

Fase correctiva: su objetivo es conseguir salud oral mediante el manejo interdisciplinario. Aquí se realiza terapia periodontal quirúrgica como raspado y alisado radicular a campo abierto, gingivectomía, gingivoplastia, frenillectomía, regeneración ósea, alargamiento de corona clínica, implantes, cirugía mucogingival, entre otros.

Fase mantenimiento: las visitas de mantenimiento cada tres meses forman la base de un programa de prevención a largo plazo. Su objetivo es prevenir la incidencia de factores locales, preservar la salud periodontal lograda como resultado del tratamiento integral.

\section{RESULTADOS}

De 1731 historias clínicas revisadas, se encontró que solo 171 (9,9\%) cumplían con los criterios de inclusión para este estudio, por las razones que se describen en la figura 1.

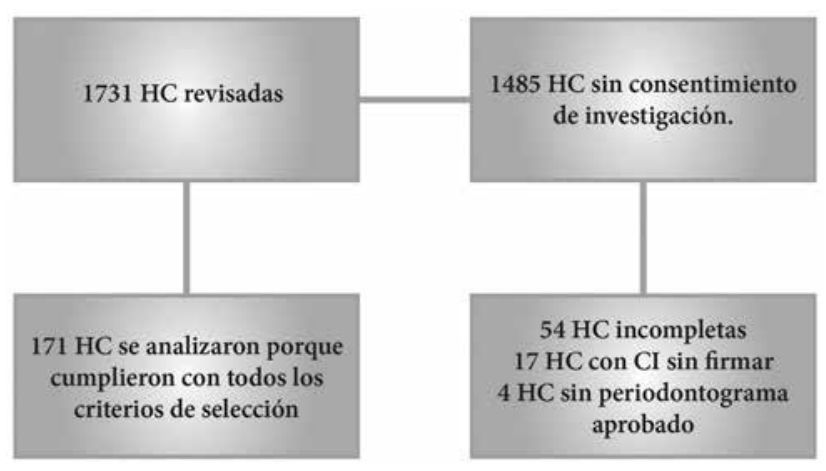

Figura 1. Diagrama de flujo que explica la inclusión de historias clínica en el estudio. HC: Historia clínica, CI: Consentimiento informado de investigación. 
Se encontró que de 171 historias clínicas que se tuvieron en cuenta para el estudio, por cumplir con todos los criterios de selección, 104 (61\%) eran de mujeres con un promedio 41,3 años, y 67 (39\%) eran de hombres con un promedio de 35,6 años. El participante con menor edad tuvo 16 años y el de mayor edad 78 años. Se destaca una muestra de la población sana en relación con el $86,6 \%$ de historias clínicas en que no se refieren enfermedades sistémicas (Tabla 1).

Se observó una tendencia de incumplimiento en todas las fases del tratamiento periodontal. Para las fases de urgencia y sistémica existe alto porcentaje en la muestra de historias clínicas estudiadas de no requerimiento.
Dentro de las fases que requieren o no de su cumplimiento; la fase de urgencia, evidenciada por dos personas, solo una de estas cumplió; en la fase sistémica, el 69,6\% asistió a la consulta para el tratamiento periodontal y para la fase correctiva, se presentó un alto nivel de incumplimiento del 78,9\%.

Seguidamente, se describe el nivel de cumplimiento de fases requeridas; en similitud, la fase higiénica, reevaluación y mantenimiento presentaron niveles altos de incumplimiento: $57,3 \%, 98,8$ y $99,4 \%$, respectivamente, siendo esta última la más alarmante (Tabla 2).

Tabla 1. Descripción de la muestra

\begin{tabular}{lccc}
\hline Característica & $\begin{array}{c}\text { Mujeres n (\%) } \\
\mathbf{1 0 4}(\mathbf{6 0 , 8})\end{array}$ & $\begin{array}{c}\text { Hombres n (\%) } \\
\mathbf{6 7}(\mathbf{3 9 , 2})\end{array}$ & $\begin{array}{c}\text { Total n (\%) } \\
\mathbf{1 7 1}(\mathbf{1 0 0})\end{array}$ \\
\hline Semestre que brinda la atención & & & \\
Sexto & $21(12,3)$ & $15(8,8)$ & $36(21,1)$ \\
Séptimo & $53(31,0)$ & $38(22,2)$ & $91(53,2)$ \\
Octavo & $11(6,4)$ & $5(2,9)$ & $16(9,4)$ \\
Noveno & $6(3,5)$ & $3(1,8)$ & $9(5,3)$ \\
Décimo & $13(7,6)$ & $6(3,5)$ & $19(11,1)$ \\
Clínica & & & $168(98,3)$ \\
Integral del adulto & $102(59,7)$ & $66(38,6)$ & $3(1,8)$ \\
Profundización rehabilitación & $2(1,2)$ & $1(0,6)$ & $53(31,0)$ \\
Diagnóstico periodontal & & & $21(12,3)$ \\
Gingivitis APB & $35(20,5)$ & $18(10,5)$ & $15(8,8)$ \\
Gingivitis APBPD & $15(8,8)$ & $6(3,5)$ & $29(17,0)$ \\
Periodontitis crónica leve & $6(3,5)$ & $9(5,3)$ & $29(17,0)$ \\
Periodontitis crónica moderada & $18(10,5)$ & $11(6,4)$ & $24(14,0)$ \\
Periodontitis Crónica Severa & $18(10,5)$ & $11(6,4)$ & $14(8,2)$ \\
Recesiones Gingivales & $12(7,0)$ & $12(7,0)$ & $2(1,2)$ \\
Compromiso Sistémico & & & $148(86,6)$ \\
\hline Hipertensión arterial & $10(5,9)$ & $4(2,3)$ & $7(4,1)$ \\
\hline Diabetes tipo I, II & $2(1,2)$ & $0(0,0)$ & \\
No refieren & $87(50,9)$ & $61(35,7)$ & $2(1,2)$ \\
\hline Otras & $5(2,9)$ & & \\
\hline & & & \\
\hline
\end{tabular}

Tabla 2. Cumplimiento de fases a criterio del odontólogo dado el curso de la enfermedad periodontal

\begin{tabular}{lccc}
\hline Fases & $\begin{array}{c}\text { Cumple } \\
\mathbf{n}(\%)\end{array}$ & $\begin{array}{c}\text { No cumple } \\
\mathbf{n}(\%)\end{array}$ & Total \\
\hline Fases requeridas según el criterio del odontólogo & & & \\
Urgencia & $1(50 \%)$ & $1(50 \%)$ & 2 \\
Sistémica & $16(69,6 \%)$ & $7(30,4 \%)$ & 23 \\
Correctiva & $30(21,1 \%)$ & $111(78,9 \%)$ & 142 \\
Fases requeridas en todos los casos & & \\
Higiénica & $73(42,7 \%)$ & $98(57,3 \%)$ & 171 \\
Reevaluación & $2(1,2 \%)$ & $168(98,8 \%)$ & 171 \\
Mantenimiento & $1(0,6 \%)$ & $170(99,4 \%)$ & 171 \\
\hline
\end{tabular}




\section{DISCUSIÓN}

El presente estudio tuvo como objetivo determinar el nivel de cumplimiento de las fases del tratamiento periodontal en pacientes que asistieron a las Clínicas Odontológicas de la Universidad Santo Tomás Campus Floridablanca y Bucaramanga, en razón a que no había estudios descriptivos que permitieran conocer la realidad del cumplimiento, dada la importancia, como se menciona anteriormente, para el éxito en el tratamiento periodontal.

Los resultados de 171 historias clínicas analizadas, correspondieron en su mayoría a mujeres que consultaron por enfermedades periodontales. Para este estudio, los eventos identificados fueron gingivitis asociada a placa bacteriana, gingivitis asociada a placa bacteriana en un periodonto disminuido, periodontitis crónica leve, moderada y severa, finalmente, deformaciones mucogingivales y condiciones alrededor del diente: tipo recesiones gingivales.

Checchi y colaboradores, señalan que el porcentaje de mujeres que se hacen tratamiento periodontal es superior al de los hombres, y refiere que no se hallan diferencias estadísticamente significativas en cuanto al género en el cumplimiento de las citas de mantenimiento ${ }^{11,12}$.

El promedio de edad de las historias clínicas de pacientes con enfermedad periodontal analizadas para este estudio fue de 39 años (DS $\pm 14,8$ ); al respecto otros estudios señalan que los pacientes de menos de 40 años tienden a ser menos cumplidores y los menores de 20 años cumplen menos visitas al tratamiento periodontal de soporte. Parece haber una correlación entre la edad y cumplimiento, de manera que a medida que aumenta la edad tiende a aumentar el cumplimiento de las citas; solo en el estudio de Checchi et al. se muestra que los pacientes jóvenes cumplen mejor las citas para tratamiento periodontal ${ }^{11}$.

Al contrastar los hallazgos del nivel de cumplimiento por fases en esta investigación, el nivel de cumplimiento de fases requeridas fue bajo. El incumplimiento para la fase higiénica, reevaluación y mantenimiento con el 57,3\%, 98,8 y 99,4\%, respectivamente, siendo esta última la más preocupante. La fase sisté- mica presentó el mayor nivel de cumplimiento con el $69,6 \%$. Al realizar el acercamiento con la literatura, es evidente que existen múltiples publicaciones que tratan sobre el cumplimiento de las citas en medicina, pero en odontología tenemos poca información y aún menos cuando se relaciona con el cumplimiento con la periodoncia.

Wilson y colaboradores, en su estudio con $961 \mathrm{pa}-$ cientes (53\% mujeres, $47 \%$ varones) todos con enfermedad periodontal crónica, que se clasificó en leve, moderada y grave, obtuvieron resultados donde solo el 16\% cumplieron el programa de mantenimiento de manera completa. El 49\% cumplieron de manera irregular y el $34 \%$ no volvieron a ningún mantenimiento. Estos resultados sugieren la existencia de una diferencia entre las visitas de mantenimiento ideales con las visitas de mantenimiento reales ${ }^{13}$.

Similar a lo encontrado por Pujol (2012) en su estudio que refiere la ocurrencia de un incumplimiento de $71,5 \%$ en la fase de mantenimiento ${ }^{14}$, en nuestro estudio este incumplimiento fue del $99 \%$ en dicha fase. De otro lado, se observó que hubo mayor cantidad de pacientes mujeres que hombres y la principal patología periodontal encontrada fue la periodontitis crónica moderada; lo anterior difiere de esta investigación donde la mayor frecuencia de consulta se debió a la gingivitis asociada a placa bacteriana (31\%), seguido por la periodontitis crónica (17\%). Lo encontrado en este estudio es consistente con los resultados de la presente investigación, en relación con las altas cifras de incumplimiento en la fase de mantenimiento ${ }^{14}$.

Desde el punto de vista etiopatogénico, el objetivo clave del tratamiento periodontal consiste en evitar que el proceso de la enfermedad vuelva a iniciar, por tanto, se plantea en mantener o establecer un equilibrio entre los factores patogénicos y la resistencia del hospedador ${ }^{15}$. Como consecuencia, la incidencia de las enfermedades periodontales debería reducirse. En concordancia con el tratamiento activo como el mantenimiento periodontal eficaz en el control de la actividad de la enfermedad periodontal, en la disminución de la destrucción periodontal y en la reducción de la mortalidad dentaria ${ }^{16}$.

La literatura demuestra la gran importancia de realizar las visitas de mantenimiento periodontal para 
el éxito a corto y largo plazo del tratamiento periodontal ${ }^{17-22}$. Actualmente, se considera como factores principales del fracaso del tratamiento periodontal, el mal control de la placa bacteriana por parte del paciente y el abandono de las visitas de mantenimiento ${ }^{23}$. Por lo tanto, es indispensable la colaboración del paciente en el cumplimiento de las visitas de soporte periódico periodontal.

Finalmente, el Estudio Nacional de Salud Bucal (ENSAB II) realizado entre 1977-1980 presentó la mayor prevalencia de enfermedad periodontal con un 95\%, mientras que hubo una notable disminución en el ENSAB III con una prevalencia del 50\%. Con respecto al ENSAB IV se presentó nuevamente una alta prevalencia de enfermedad periodontal con un $72 \%$ en el periodo 2013-201424,25. Según Duque y cols., la alta prevalencia de enfermedad periodontal a nivel mundial puede atribuirse en muchos casos al poco interés en la promoción y prevención de la salud oral $^{26}$; por ende, la alta prevalencia de enfermedad periodontal debería enmarcar la importancia del cumplimiento al tratamiento, pero lamentablemente no es así. Por lo tanto, se fundamenta, al conocer la realidad de la falta de cumplimiento de las fases del tratamiento periodontal, implementar estrategias, protocolos o intervenciones educativas desde el primer contacto entre el profesional-paciente. Es oportuno y crucial el brindar el tiempo durante la consulta para las explicaciones del profesional y para dar mayor importancia al hecho de cumplir las citas para tratar la patología periodontal.

Una de las limitaciones del presente estudio fue la falta de diligenciamiento del consentimiento informado lo que redujo de manera considerable el número de historias clínicas a las que se pudo acceder para generar los datos presentados. No obstante, este trabajo aporta información relevante respecto al nivel de cumplimiento en las fases del tratamiento periodontal y por ello se constituye en un material importante para tomar decisiones clínicas.

\section{CONCLUSIÓN}

El nivel de cumplimiento de las fases higiénica, de reevaluación y de mantenimiento del tratamiento periodontal fue muy alto $(57,3 \%, 98,8$ y $99,4 \%$ respectivamente), siendo esta última la más preocupante.
Probablemente, las causas de este incumplimiento sean debido a estrategias de educación débiles y poca motivación para el paciente. Por lo tanto, se hace pertinente la creación, rediseño y fortalecimiento de intervenciones en la consulta para estimular al paciente a que se adhiera al tratamiento y asista a todas las fases de reevaluación y mantenimiento.

\section{BIBLIOGRAFÍA}

1. Carvajal P. Enfermedades periodontales como un problema de salud pública: el desafío del nivel primario de atención en salud. Rev Clín Periodon Implantol Rehabil Oral. 2016;9(2):177-183.

2. Arteaga A. Desarrollo y evaluación de una aplicación informática para registrar el P.S.R, índice de placa, periodontograma de los pacientes atendidos en la Clínica Odontológica Integral de la Universidad de las Américas, Quito-Ecuador. Universidad de las Américas; 2016.

3. Orozco A, Raigosa M, Ceballos A, Quintero P. La terapia periodontal regenerativa. CES Odontol. 1997;10(1): 1-26.

4. Ferro M, Gómez M. Fundamentos de la Odontología. 2da. ed. Bogotá; 2007.

5. Lindhe J, Lang N, Karring T. Periodontología clínica e implantología odontológica. 5ta. ed. Buenos Aires: Médica Panamericana; 2008.

6. Tugnait A, Clerehugh V, Hirschmann PN. Use of the basic periodontal examination and radiographs in the assessment of periodontal diseases in general dental practice. J Dentis. 2004;32(1):17-25.

7. Newman M, Takei H, Klokkevold P, Carranza F. Periodontología Clínica de Carranza. 11va. ed. Amolca; 2014.

8. Ramírez J, Contreras A. ¿Se debe considerar a la enfermedad periodontal un problema de salud pública en Colombia? Colomb Méd. 2009;38(3):181-184.

9. Manjon M, Martínez O. The chi-squared goodness-of-fit test for count-data models. Stat Journ. 2014;14(4):798-816.

10. Armitage GC. Development of a classification system for periodontal diseases and conditions. Annals periodontol. 1999;4(1):1-6.

11. Checchi L, Pelliccioni GA, Gatto MRA, Keiescian L. Patient compliance with maintenance therapy in an Italian periodontal practice. J Clin Periodontol. 1994;21(5):309-312. 
12. Novaes Jr AB, Novaes AB. Compliance with supportive periodontal therapy. Part 1. Risk of non-compliance in the first 5-year period. J Periodontol. 1999;70(6):679-682.

13. Wilson Jr TG, Glover ME, Schoen J, Baus C, Jacobs T. Compliance with maintenance therapy in a private periodontal practice. J Periodontol. 1984;55(8):468-473.

14. Pujol M. Estudio sobre el cumplimiento (valoración de factores de riesgo) en las visitas de soporte periodontal de pacientes con patología periodontal [tesis]. Universidad de Valencia, España; 2012.

15. Botero J, Bedoya E. Determinantes del diagnóstico periodontal. Rev Clín Periodon Implantol Rehabil Oral. 2010;3(2):94-99.

16. Bascones A, Figuero E. Las enfermedades periodontales como infecciones bacterianas. Med Oral Patol Oral Cir Buc. 2004;9:92-107.

17. Carvalho V, Okuda O, Bernardo C, Pannuti C, Georgetti $M$, De Micheli $G$ et al. Compliance improvement in periodontal maintenance. J App Oral Scien. 2010;18(3):215-219.

18. Novaes Jr AB, Novaes AB. Compliance with supportive periodontal therapy. Part 1. Risk of non-compliance in the first 5-year period. J Periodontol. 1999;70(6):679-682.

19. Mendoza AR, Newcomb GM, Nixon KC. Compliance with supportive periodontal therapy. J Periodontol. 1991;62(12):731-736.

20. Loesche WJ, Grossman N, Giordano J. Metronidazole in periodontitis (IV). The effect of patient compliance on treatment parameters. J Clin Periodontol. 1993;20(2):96-104.
21. Novaes Jr AB, Novaes AB, Bustamanti A, Villavicencio B JJ, Muller E, Pulido EJ. Supportive periodontal therapy in South America. A retrospective multi-practice study on compliance. J Periodontol. 1999;70(3):301-306.

22. Wilson Jr TG, Glover ME, Schoen J, Baus C, Jacobs T. Compliance with maintenance therapy in a private periodontal practice. J Periodontol. 1984;55(8):468-473

23. Lafaurie G, Contreras A, Barón A, Botero J, Mayorga-Fayad I, Jaramillo A et al. Demographic, clinical, and microbial aspects of chronic and aggressive periodontitis in Colombia: a multicenter study. J Periodontol. 2007;78(4):629-639.

24. IV Estudio Nacional de Salud Bucal, ENSAB IV [Internet]. Bogotá D.C. Ministerio de Salud. [Actualización: 2013-2014; Citado: 2016 Abr 30]. Recuperado a partir de: http://www.minsalud.gov.co

25. III Estudio Nacional de Salud Bucal, ENSAB III [Internet]. Bogotá D.C. Ministerio de Salud. [Actualización: 1999; Citado: 2015 Nov 23]. Recuperado a partir de: http://www.minsalud.gov.co

26. Duque A, Cuartas C, Muñoz C, Salazar C, Sánchez Y. Nivel de conocimiento sobre enfermedad periodontal en una muestra de empleados en Medellín. Rev CES Odontol. 2011;24(2):43-47. 